\title{
Location Awareness Impact on Long Term Evolution (LTE) System
}

\author{
Muaz Saleem \\ ICT, UET Peshawar \\ PAKISTAN
}

\author{
Mobasher Ali \\ MSc Computer \\ Engineering
}

\author{
Asad Ali \\ LUMS, Lahore \\ PAKISTAN
}

\begin{abstract}
Packet scheduling algorithms play an important role in determining throughput performance of LTE system. A stationary user with poor CQI (channel quality indicator) has smaller throughput and present scheduling algorithms are unable to address this issue. In this paper, a new scheduler technique is proposed which enhances throughput and fairness in LTE system for stationary as well as mobile users. This paper takes up preexisting scheduling algorithms and modifies them to schedule users according to a different criterion like fairness and Channel Quality Indicator. A hybrid of Round Robin and Best CQI techniques has been proposed for scheduling of the users which produces increased throughput for different SNR values simulated against Pedestrian and Vehicular moving models. The proposed model is very effective for stationary users, increasing throughput up to $500 \%$ and has been verified through MATLAB simulations.
\end{abstract}

\section{Keywords}

LTE, Radio Resource Management (RRM), Round Robin Scheduler, Best CQI Scheduler.

\section{INTRODUCTION}

Wireless technologies are usually grouped under various heading of 'generations'. Each generation depicts a discrete level of maturity of standards and technical details. Still there is no strict demarcation between each generation. To understand this concept one needs to segregate telecommunication paradigm in certain layers, vis. Access, Core and Transportation. Presently, engineers are specializing in each layer and hastening the process of evolution for each stage. Core engineers have been able to rapidly transform old circuit switched systems in packet switching. Transmission engineers have been able to transform systems to advance optical fiber networks based on dense wavelength division multiplexing (DWDM), to meet high data speed demands. On the 'Access' side, there are two main categories which overshadow the telecom model, vis. Wired and Wireless User Interface. These interfaces are rapidly advancing on the face of innovative access technologies like Code Division Multiple Access (CDMA) and Orthogonal Frequency-Division Multiple Access (OFDMA). The terminologies like 3rd and 4th generation systems necessarily include access technologies having high data rate user interface. These interfaces are enabled by exploiting Shannon Capacity limit through technologies like MIMO-OFDM (as in WiMAX) and WCDMA (as in UMTS). [1]

Since Core and Transport layers are based on Ultra High Switching systems and optical fibers, it is imperative to enable Access layer at par with the other two. Various technologies were developed to address the problem of high data rate access. Users are disposed to get services that may provide them with live streaming and other real time services. In case of GSM (2G) standards data rate never hoped a sum of 180 kbps on minimum concurrence. There were other issues like small scale fading, large scale fading, inter symbol interference and noise of various types. The seriously impaired second generation telephony had a voracious appetite of newer standards of communication. IP based switching rendered circuit switching obsolete and demands of high capacity Access technology arose [2]. CDMA had an advantage of better spectrum usage allowing more users to access the media. Users operating simultaneously within a shared frequency band differentiated each other on the base of code (orthogonal code) [3]. Thus the issue of interference and frequency reuse pool is mitigated in a distinct way. The digital data rate of CDMA system varied from 4.8 to $14.4 \mathrm{kbps}$ where as its next evolution i.e. CDMA-Two/ IS-95b supported data rate of as high as $115.2 \mathrm{kbps}$ [3].

Long Term Evolution (LTE) is the brand name of 3GPP's present shape known to European-engineers as 4G. As a result of ITU moots on 4G/3GPP/3GPP2/LTE/UMB following requirements have been set [4]. The ambition as noted from credible source is the achievement of $326 \mathrm{Mbps}$ in downlink (peak) speed, whereas 86 Mbps of peak uplink speed. The bandwidth requirement for utilization in MIMO system is of $20 \mathrm{MHz}$. Also the mandate for round trip latency between user module (mobile phone) and Base Station (BS) is set at 10 milliseconds [5].

The throughput of a UE depends on the different factors and scheduling algorithm is a major one. Robin scheduling techniques treats each user equally without taking CQI into account and, thus, reduces throughput but maintains fairness. CQI based scheduling favors user with high SNR, increasing channel capacity but reducing fairness. Proportional fair scheduling provides balance between maximum cell throughput and fairness. Proposed scheduling technique is a kind of proportional fair scheduling with increased throughputs that operates between the Best CQI scheduler and the Round Robin scheduler.

This paper is organized in five sections: Second section provides background and research being done regarding LTE system. Third section discusses management of system resources and existing scheduling algorithms in this regard. Fourth section talks about proposed hybrid scheduling algorithm and displays simulations results. Finally, in fifth section, conclusions are drawn.

\section{BACKGROUND OF LTE}

Before getting a direct interaction with LTE systems, it is necessary to refer a few system diagnostics. In order to achieve objectives of this work, it is important to study already available research, especially work done on the user channel (access systems). David Astely, Erik Dahlman and Stefen Parkvall at Ericsson research center came up with a white paper in 2009. It was presented in a workshop that aimed at highlighting the progress of $3 \mathrm{GPP}$ on Long Term 
Evolution. Another paper by Sundeep Palat and Philppe Godin, in 2009 The LTE Network Architecture- Strategic White Paper was also presented during later years which provided with the details of Release 8 of the specifications [6]. Main emphases of these papers were:
a. Network Architecture
b. Interfaces that enable LTE networks
c. Optimization and Efficiency of the system

Main aspect of LTE is that in comparison to erstwhile cellular models, LTE is a complete packet switched. A seamless IP service is sanctioned and exceptionally guaranteed from Packet Data Network (PDN) to user Equipment (UE). Here it is important to mention that there are two main sections of research study. LTE is based on Universal Mobile Telecommunication System (UMTS) Radio Terrestrial Access Network (UTRAN), thus there are two main research areas according to the evolution perspective:

a. System Architecture Evolution which has Evolved Packet Core Network (CN), NonRadio Part

\section{b. Evolved UTRAN (Access The Radio Part)}

Note that these two aspects are collectively called Evolved Packet System (EPS), communication/ data transfer session established is called EPS bearer. This bearer is actually IP traffic from a gateway in the packet data network PDN to user equipment UE [7].

In Figure 1, EPS network elements are shown. EPS provides UE an IP for its connectivity to EPC so that it can communicate with other VoIP users and access the Internet. Multiple EPS bearers can be established for a single user and each of these EPS bearers has a specific QoS priority according to the type of service. A VoIP bearer would have a distinct priority as it is different from FTP session or a web browser. The network should also be secure and must have sufficient security against black hat attacks.

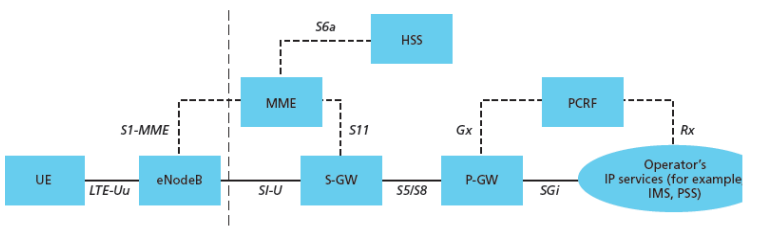

Figure 1: EPS Network Elements

Functionality of each module and its subsections is briefly described below. Note that the terminologies define both the EUTRAN and EPS of SAE which also includes the core network $(\mathrm{CN})$ of EPS. A brief on the overall architecture of EPS is discussed in this section which helps in segregating the protocols that distinguish radio network from non-radio network (SAE EPC). Like BTS (Base Trans-receiver Station) and BSC (Base Controller Station) form a dendrites topology which were replaced by NodeB and RNC (Radio Network Controller) in UMTS. Later the RNC functionality was dissolute in Mobility Management Element (MME) and merged in non-radio part of the EPS (but not in EPC subsection). The evolved NodeB (eNodeB) is a step forward which was incorporated in LTE access systems [8]. UEs are connected to eNodeB through wireless interface named LTRUu but the mobility management utility in LTE is provided by
S1-MME link (Management signaling link between EPS and eNodeB, the E-UTRAN). At this point of discussion on LTE, it is time to start off with the actual area of research which is on Radio Resource Management (RRM). As previously referred, in EUTRAN (NAS and AS protocols).

\section{RADIO RESOURCE MANAGEMENT}

RRM is one of the main areas of research for engineers who try to make network robust and resilient. RRM consists of a number of algorithms that deal with various system resources. RRM functions are performed by Physical, Data Link and Network layer. However, the functionality of access part deals with the first two layers.

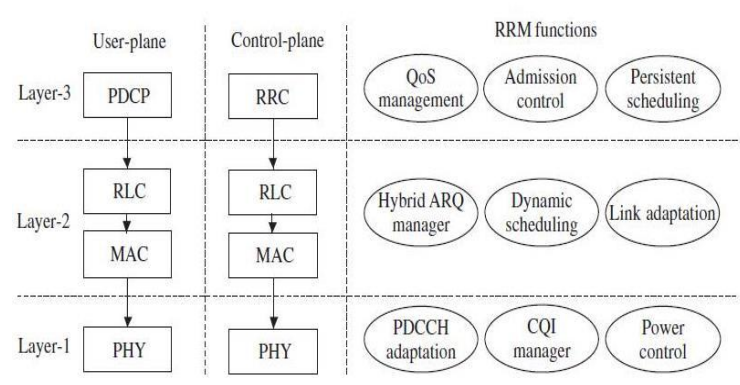

Figure 2: Radio Resource Management

\subsection{Functionalities}

Channel Quality Indicator (CQI) Manager is a Layer 1 Protocol which has the downlink status report and its counterpart is Sounding Reference Signals (SRS) in the uplink direction. Each CQI and SRS is used by eNB for the scheduling and link adaptation processes at Layer 2. Here the semi persistent scheduling will not be discussed as it involves the core part [8]. If a new UE enters or gets activated into a cell, it sends a request, Admission Control decides through an algorithm whether to accept or reject UE's request for EPS bearer. The algorithm of Admission Control keeps into account the availability of bearers, QoS, priority level and certain other parameters of session control in order to allocate a fresh EPS bearer to the new UE. In this algorithm (QoSbased-AC) the QoS factor is of prime importance and $\mathrm{AC}$ (Admission Control) only allocates a bearer, it is able to maintain QoS requirements for that UE. However, AC is vendor dependent and the brand name gives an epithet for the minimum QoS it guarantees. Radio Resource Management at Layer 2 is done through Packet Scheduler every TTI, which has following functions:

\section{a. Allocation of Physical Resource Blocks (PRB) \\ b. Modulation \\ c. Coding Scheme}

PDCCH is used for intimation of the above three parameters to the user. Scheduling, thus, can be defined as a protocol which is used to maximize the cell capacity keeping in view the QoS requirements (best effort bearers). The decisions regarding these parameters as per user requirements and is matured with every TTI with the help of several data flows.

Each data flow (user plane and control plane) is identified allotting it a 5-bit Logical Channel Identifier (LCID). Transport Block Size (TBS) is separate for each user and on the base of this TBS; MAC Protocol takes decision for the transfer of data from a single LCID. Another function of packet scheduler is its interaction with Hybrid ARQ Manager which carries out retransmission [9]. HARQ manager, 
precisely, has a function of scheduling retransmission. In case of downlink, for each code-word there are 8 channels. This means that the scheduler can dynamically schedule HARQ retransmission. The transmission can occur in time and frequency domains as per vendor designation. After every sub-frame (TTI) the scheduler decides to transmit using HARQ technique.

Link Adaptation gives information to the scheduler so that modulation technique and coding scheme can be decided for an UE. The adaptation depends upon PRBs related to the target UE. The most important dependency of LA unit of Layer 2 is the feedback from the UEs. The feedback is conveyed via CQI (discussed earlier). Link Adaptation can be carried out with the help of a separate loop link between UE and eNB. In such a case HARQ detention or permission can be based on previous transmission. This method is used in HSDPA.

\subsection{Scheduling}

In this process, users are selected for Physical Resource Block (PRB) allocation. Different algorithms are used for taking scheduling decisions. The basic Packet Scheduling (PS) techniques that are currently being deployed in LTE $4 \mathrm{G}$ are:

\section{a. Frequency Domain Packet Scheduling \\ b. Time Domain Packet Scheduling \\ c. Combined Time and Frequency Domain PS \\ d. Packet Scheduling with MIMO (OFDM)}

Details of these chief scheduling techniques are given below which discuss the main principles of the scheduling techniques. However, the resources and algorithms used for provision of these resources, today, are being used in a more complex manner. Scheduling is done for only those users which have high channel quality existing on Physical Resource Block (PRB). Those users which are in deep fades are excluded from this scheduling process. High Frequency Domain Packet Scheduling (FDPS) are expected to be gained due to inherent design of this type of scheduling technique. A typical case of scheduling involves less channel bandwidth and high system bandwidth.

Time domain scheduling also provides multi-user diversity gains. But in the case of time domain scheduling, gain depends upon amount of fading and speed of fading. The deep fades are not included in the scheduling process which excludes them from the process of scheduling till their reengagement request into the system. Through this method Time Domain Packet Scheduling (TDPS) spectral efficiency can be increased by inclusion of the process of frequency usage. A condition of fractional load mode can occur if eNB has small data to be transmitted. A limited number of PRBs are required for the process and rest of the PRBs are muted. In such condition TDPS uses PRBs with highest channel quality which is based on CQI feedback. Another technique in packet scheduling is the employment of both time and frequency domain packet schedulers. When UEs are selected a priority metric is assigned to them in the PRB and the control channel schedule performs an evaluation. If transmission resources are fine, PDCCH is used as an authenticator for the last step which is FDPS. Configuration can be done at FDPS stage for prioritizing of the pending HARQ retransmissions [10]

\subsection{Round Robin Technique}

The PRB allocated to each UE in an area is equal. One after another UE with CQI information is inducted in the system. However, CQI is not a metric that is taken into account to have them inducted for PRB allocation. Hence all the users are equally opportune for scheduling. This method is known as Round Robin [11]. Main advantage of this type of scheduling technique is ease of implementation; however, $\mathrm{CQI}$ is not taken into account to set any performance criteria. This not only degrades the throughput but also reduces the QoS and System Capacity. CQI is an important performance metric that is used for equal throughput among participating UEs and feedback mechanism.

\subsection{Best Cqi Technique}

Best CQI Scheduling is a more enhanced technique for increasing system capacity of 4G LTE system. Signal to Noise Ratio (SNR) takes into account CQI information from UEs. On the base of CQI, PRB is assigned to UE. eNB must have CQI for Max SINR implementation. Good channel capacity guarantees better CQI which in turn gets priority for a set of UEs. GBR is ensured with high QoS in this type of scheduling [12]. In this type of scheduling the UEs which are far from eNB and cannot be guaranteed QoS are not inducted to scheduling.

\subsection{Proportional Fair Technique}

On the base of best relative channel quality, PRBs are assigned to UEs. CQI plays an important role in selection of UEs, as seen in case of SNIR. Another metric is level of fairness which differentiate this type of scheduling with SINR. Due to this feature this type of scheduling is called Proportional Fair Scheduling. Main aim of this method is to strike a balance between Maximum Cell Throughput and Fairness [13]. With this way users are able to achieve Minimum Quality of Service. There are a number of PF scheduling versions. The hybrid technique is proposed in order to increase system throughput, a design which makes use of prevalent scheduling techniques at the same time is being proposed for testing.

\section{SYSTEM DESIGN AND SIMULATION}

The techniques which are suitable for mobile UEs and currently prevalent in the deployed systems are Round Robin and Best CQI Scheduling. As discussed in previous chapter on the basis of CQI one could initiate Round Robin method and in a loop, selections can be made. For users which are stationary, have better channel stat information (CSI), thus giving better CQI The model is based on the concept of merging Round Robin and CQI based Schedulers. This helps in commissioning UEs which are stationary at first place. The mobile users which have less CSI, then have to wait for next time slot. The Schedulers are implemented in Matlab. 


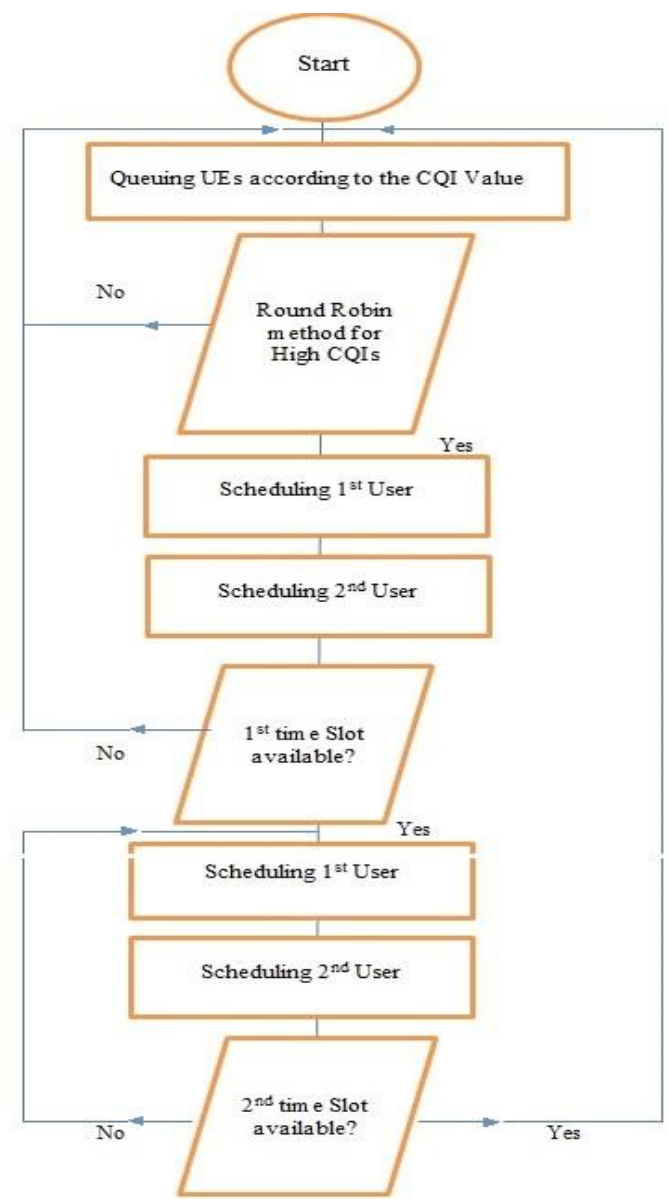

Figure 3: Flow Chart of Proposed Model

In order to find a trade-off between throughput and fairness, a new scheduling algorithm is proposed that operates between the Best CQI scheduler and the Round Robin scheduler. The new scheduling algorithm will result in an acceptable throughput and provides some fairness between users. A physical resource block (PRB) is the smallest element of resource allocation assigned by the scheduler. In first step, the queue is set up on the basis of best CQI. For this queue round robin technique is deployed for scheduling the users. The round robin technique is applied for best CQI queue as far as 1 st time slot of each sub-frame is available. When 1st time slot is not available, queuing is again done on the basis of best CQI values. Similarly queue on the basis of best CQI is set up in case of availability of 2 nd time slot in each sub-frame. In this way, thus a compromise between the fairness and the throughput can be reached.

\subsection{Simulation Parameter}

Simulation parameters over which traditional Round Robin Scheduling is being tested are given in the table 4.1. Note that the Round Robin only schedules UEs with CQI value set at 7. Other parameters are the same and are set for a typical scenario. For both the mobile and stationary UEs the code is executed.

After implementing the above mentioned scheduler, modified algorithm is executed. The parameters are given below whereas the test bed is the same (link level simulation of LTE).
Table 1: Simulation parameters for round robin technique

\begin{tabular}{|l|c|}
\hline \multicolumn{1}{|c|}{$\begin{array}{c}\text { Simulation Parameters and } \\
\text { Type }\end{array}$} & Scenario Value/ Type \\
\hline Number of Base Station(s) & 1 \\
\hline User Equipment & 2 \\
\hline $\begin{array}{l}\text { Channel Type (Mobile and } \\
\text { Stationary) }\end{array}$ & Vehicle A/Pedestrian B \\
\hline $\begin{array}{l}\text { Scheduling Algorithm (With } \\
\text { CQI) }\end{array}$ & $\begin{array}{c}\text { Round Robin with } \\
\text { CQI=7 }\end{array}$ \\
\hline $\begin{array}{l}\text { Simulation Length (Sub- } \\
\text { Frame) }\end{array}$ & 100 \\
\hline Bandwidth-Set & $1.4 \mathrm{MHz}$ \\
\hline Transmission Scheme & $\begin{array}{c}\text { Single Input Single } \\
\text { Output }\end{array}$ \\
\hline
\end{tabular}

Table 2: Simulation parameters for Proposed Model

\begin{tabular}{|l|c|}
\hline \multicolumn{1}{|c|}{$\begin{array}{c}\text { Simulation Parameters and } \\
\text { Type }\end{array}$} & Scenario Value/ Type \\
\hline Number of Base Station(s) & 1 \\
\hline User Equipment & 2 \\
\hline $\begin{array}{l}\text { Channel Type (Mobile and } \\
\text { Stationary) }\end{array}$ & Vehicle A/Pedestrian B \\
\hline $\begin{array}{l}\text { Scheduling Algorithm (With } \\
\text { CQI) }\end{array}$ & $\begin{array}{c}\text { Proposed (CQI } \\
\text { dependent and RR) }\end{array}$ \\
\hline $\begin{array}{l}\text { Simulation Length (Sub- } \\
\text { Frame) }\end{array}$ & 100 \\
\hline Bandwidth-Set & Single Input Single \\
Transmission Scheme & Output \\
\hline
\end{tabular}

\subsection{Simulation Results}

The results of the above mentioned simulation model will be discussed here. The simulator values remained the same while both the algorithms were replaced in the simulator to be tested. In old scheduling (simple round robin) technique different scenarios for SISO and MIMO channel models were simulated for stationary user. The graph suggests that the throughput increased substantially in the use of MIMO channel. Round Robin scheduling technique is used for simulation. For lower SNR values, throughput is also very low for all type of channels (SISO and MIMO). For very good channel and higher SNR values, throughput is boosted and went up to 50 Mbps using MIMO $4 \times 4$ channel.

The comparative analysis is done for stationary users, the moving model PedB is implemented for simulation of both round robin and proposed schedulers. The best channel MIMO $4 \times 4$ is discussed being best throughput generator in both cases. At the SNR value $10 \mathrm{~dB}$, proposed technique has generated double throughput i.e. $20 \mathrm{Mbps}$ as compared to 10 Mbps in case of round robin scheduling. At the higher SNR 
values, the throughput gap is more evident for proposed scheduler. For instance, at $35 \mathrm{~dB}$, round robin has $15 \mathrm{Mbps}$ and proposed scheduler has more than $100 \mathrm{Mbps}$ throughput.
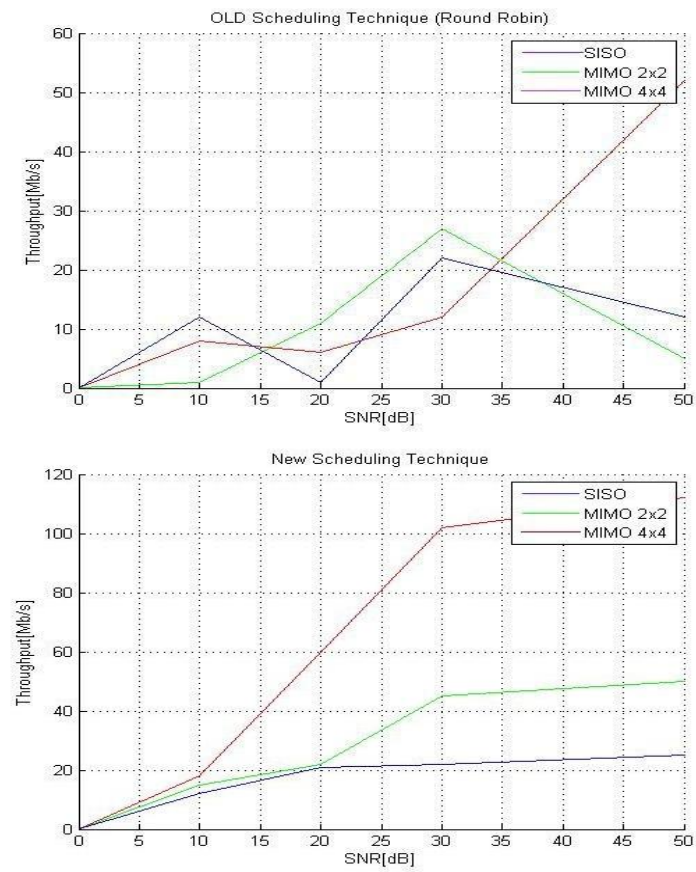

Figure 4: Comparative Analysis for Stationary Users Throughput Vs SNR

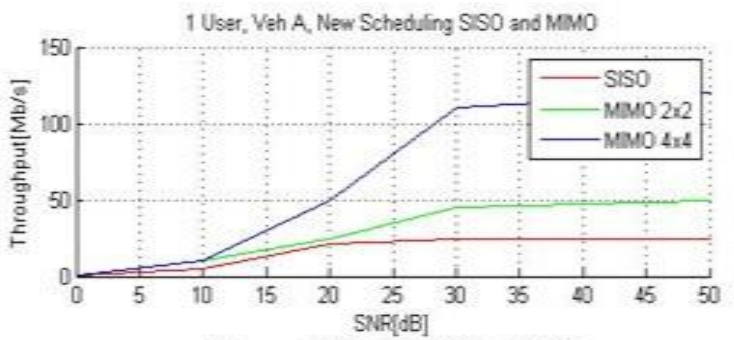

1 User, round Robin Veh A SSO and MMO

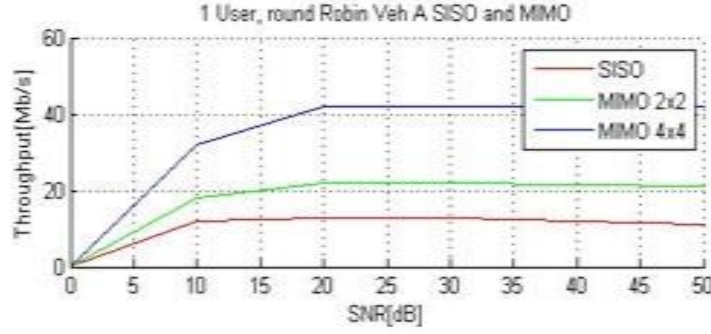

Figure 5: Comparative Analysis for Moving Users Throughput Vs SNR

The throughput trends are same for moving users as that of stationary users. The peak value of proposed model is always higher than the peak value of Round Robin scheduler for each and every value of SNR. At higher SNR, difference is more prominent and gap is also more evident for overall peak values. For instance, at $30 \mathrm{~dB}$ SNR value, new proposed scheduler has throughput of $100 \mathrm{Mbps}$ as compared to 40 Mbps in case of Round Robin for moving user as depicted in Fig. 5.

\section{CONCLUSION}

A scheduling technique which is hybrid of round robin and best CQI has been proposed in this paper. MATLAB simulations show that the proposed technique is able to double the throughput even at low SNR and has shown a huge increase of up to $500 \%$ for higher SNR in case of stationary user as shown in Fig. 4. It is important to mention here that this technique will help in segregation and prioritizing stationary users in special cases. As for example if LTE operator wants better facilitation of users who are stationary, this model will prove to be the best option. In such cases, the network resources of switching users and bearer channels are saved. Overall, the throughputs (both for mobile and stationary users) are improved as compared to Round Robin technique and fairness is also improved for proposed model. The technique can be used to optimize systems which are currently deployed by various operators. As a future work, a comparison of this proposed technique can be made with various other scheduling models.

\section{ACKNOWLEDGMENTS}

A large number of people have made possible and contributed to my work on this thesis in different ways. It is a pleasure to convey gratitude to all of them all acknowledgment. Foremost, the support, guidance and encouragement by supervisor Engr. Syed Shees Ashraf and Professor Dr. Shahzada Alamgir, help to perform at critical stages. Their professional vision and technical skills always proved helpful in problem solving and addressing issue.

\section{REFERENCES}

[1] What is 4G LTE Advanced | Long Term Evolution | Tutorial Radio-Electronics.com. (n.d.). Retrieved from http://www.radio-

electronics.com/info/cellulartelecomms/lte-long-termevolution/3gpp-4g-imt-lte-advanced-tutorial.php.

[2] Yegenoglu, F.; Alexander, R.; Gokhale, D., "An IP transport and routing architecture for next-generation satellite networks," Network, IEEE , vol.14, no.5, pp.32,38, Sep/Oct 2000 doi: 10.1109/65.87133.

[3] Ben Volkow (2013). Why LTE Needs Diameter Signaling Management. (n.d.). Retrieved from http://www.tmonet.com/voip/departments/articles/33605 6-why-lte-needs-diameter-signaling-management.htm.

[4] Ji-Hoon Yun; Myoungwon Lee; Suhan Choi, "Comparison of handover schemes for 3GPP Long Term Evolution and 3GPP2 Ultra Mobile Broadband," Personal, Indoor and Mobile Radio Communications, 2008. PIMRC 2008. IEEE 19th International Symposium, vol., no., pp.1,5, 15-18 Sept. 2008 doi: 10.1109/PIMRC.2008.4699452.

[5] Ian Poole, (2012). What is HSPA | 3G High Speed Packet Access | Radio-Electronics.com. (n.d.). Retrieved from http://www.radioelectronics.com/info/cellulartelecomms/3g-hspa/umtshigh-speed-packet-access-tutorial.php.

[6] Sundeep Palat, Philppe Godin, 2009 Handover from EUTRAN to UTRAN/GERAN CS Patent Application (Application \#20080267128 issued October 30, 2008) Justia Patents Database. (n.d.). Retrieved from http://patents.justia.com/patent/20080267128.

[7] Huang Tao, Zhang Zhijiang, Liu Yunjie, 2009. QoS Mechanism in EPS - ZTE Corporation. (2011). Retrieved fromhttp://wwwen.zte.com.cn/endata/magazine/ztecomm unications/2009year/no1/articles/200903/t20090319_170 889.html. 
[8] Samia Dardouri, Ridha Bouallegue. (2014). Comparative Study of Scheduling Algorithms for LTE Networks, World Academy of Science, Engineering and Technology International Journal of Computer, Information, Systems and Control Engineering Vol:8 No:3, 2014. Retrieved from http://waset.org/publications/9997761/comparativestudy-of-scheduling-algorithms-for-lte-networks.

[9] Rohde Schwarz. (2011). UMTS Long Term Evolution (LTE) - Technology Introduction Application Note.

[10] Joel Meza. (n.d.). LTE for UMTS - OFDMA and SCFDMA Based Radio Access | Joel Meza - Academia.edu. Retrieved from http://www.academia.edu/5300972/LTE_for_UMTS__OFDMA_and_SC-FDMA_Based_Radio_Access.
[11] Abdulhasan, M.Q.; Salman, M.I.; Chee Kyun Ng; Noordin, N.K.; Hashim, S.J.; Bin Hashim, F., "A threshold feedback compression scheme of channel quality indicator (CQI) in LTE systems," Research and Development (SCOReD), 2013 IEEE Student Conference on , vol., no., pp.181,186, 16-17 Dec. 2013 doi: 10.1109/SCOReD.2013.7002567.

[12] G. Aiyetoro, G. Giambene and F. Takawira, " A New Packet Scheduling Algorithm in Satellite LTE networks," IEEE AFRICON Conference, Mauritius, September 2013

[13] R. Almatarneh, M. Ahmed, and O. Dobre, "Performance analysis of proportional fair scheduling in ofdma wireless systems," in Vehicular Technology Conference Fall (VTC 2010-Fall), 2010 IEEE 72nd, sept. 2010, pp. $1-5$ 